\title{
Reconsolidation of a context long-term memory in the terrestrial snail requires protein synthesis
}

\author{
Tatiana H. Gainutdinova, ${ }^{1}$ Rosa R. Tagirova, ${ }^{1}$ Asja I. Ismailova, ${ }^{1}$ \\ Lyudmila N. Muranova, ${ }^{1}$ Elena I. Samarova, ${ }^{2}$ Khalil L. Gainutdinov, ${ }^{1}$ \\ and Pavel M. Balaban ${ }^{2,3}$ \\ ${ }^{1}$ Kazan Physical Technical Institute of Russian Academy of Sciences, Kazan 420029, Russia; ${ }^{2}$ Institute of Higher Nervous Activity \\ and Neurophysiology, Russian Academy of Sciences, Moscow 117485, Russia
}

\begin{abstract}
We investigated the influence of the protein synthesis blocker anisomycin on contextual memory in the terrestrial snail Helix. Prior to the training session, the behavioral responses in two contexts were similar. Two days after a session of electric shocks (5 d) in one context only, the context conditioning was observed as the significant difference of behavioral response amplitudes in two contexts. On the day following testing of context learning, a session of "reminding" was performed, immediately after which the snails were injected with anisomycin or vehicle. Testing of long-term context memory has shown that only anisomycin injections impaired the context conditioning. In control series, the snails were injected after the training session with anisomycin/saline without reminding, and no impairment of the long-term context memory was observed, while injection of anisomycin during the training session completely abolished the long-term memory. No effects of anisomycin on the short-term memory were observed. Surprisingly, injection of anisomycin after the reminding combined with reinforcing stimuli elicited no effect on the context memory. Differences between single-trial and multisession learning are discussed.
\end{abstract}

Two important features of the original accounts of associative learning (Pavlov 1927) are used again by modern scientists. First, any paradigm of associative learning implies simultaneous retaining of (1) the memory of a context (considered to be a part of declarative memory) and (2) the memory of a particular cue requiring behavioral response or a skill (named nondeclarative, cued, or procedural). Second, these two types of memory originate synergically but are independent (described originally as independent conditioned reflexes) and, as was shown recently, may have different cellular mechanisms (Balaban and Bravarenko 1993; Balaban 2002a; Debiec and LeDoux 2002; Thomas et al. 2002). We concentrated our efforts on behavioral and cellular mechanisms of procedural and contextual memories in the terrestrial snail Helix (Balaban 1993, 2002a; Balaban and Bravarenko 1993). In short, by using the paradigm leading to formation of the procedural memory (conditioned withdrawal), it was shown that activation of a single identified serotonergic cell is sufficient to trigger a long-term associative enhancement of monosynaptic input to the withdrawal interneuron in a simple network consisting of three interconnected identified cells (Balaban et al. 2004). Mechanisms of long-term synaptic changes are similar to those described in Aplysia (Antonov et al. 2003). Investigation of cellular mechanisms of context memory involved in aversive learning in Helix showed that the serotonergic modulatory neurons are the key players. These neurons respond to stimuli present in the environment ( $\mathrm{pH}$ of the substrate, temperature, tactile information, light level), and their activation corresponds to behavioral arousal of the animal (Zakharov et al. 1995; Balaban 2002b). It is still unclear whether mechanisms involved in context conditioning are similar to mechanisms of procedural memory.

\footnotetext{
${ }^{3}$ Corresponding author.

E-mail balaban@ihna.msk.ru; fax 1-617-6873051.

Article and publication are at http://www.learnmem.org/cgi/doi/10.1101/ Im.25705.
}

One of the most interesting properties of memory is that a stable long-consolidated memory can be disturbed by the same factors that can impair newly formed memories shortly after acquisition, if applied along with "reminder" cues representing a part of the learning situation (Misanin et al. 1968; Nader et al. 2000; Sara 2000). It is not understood completely whether this impairment is due to reconsolidation of a previously existing memory de novo, or to a retrieval deficit (Nader et al. 2000; Anokhin et al. 2002; Duvarci and Nader 2004). Context-specific learning and its memory have been shown in many invertebrates (Colwill et al. 1988; Balaban and Bravarenko 1993; Haney and Lukowiak 2001). Reactivation of memory interpreted as reconsolidation also was shown in invertebrates (Sekiguchi et al. 1997; Pedreira et al. 2002; Sangha et al. 2003).

Recently, effective blockade of protein synthesis by anisomycin (AN) in identified neurons of terrestrial snail Helix was demonstrated (Ghirardi et al. 2004). In the present study, we describe the effects of protein synthesis blockade on the retention of context memory and the effects of reminding in the terrestrial snail.

\section{Materials and Methods}

\section{Subjects}

In the present series of experiments, 161 adult snails Helix lucorum L. (Crimea population) weighing 30-35 g were used. The snails were kept in active state at least $2 \mathrm{wk}$ before the experiment in a wet environment and were fed usually with carrots. Two days before the training session, the experimental animals were deprived of food. Between experimental sessions, the subjects were housed in glass terrariums with food ad libitum.

Six series of experiments with yoked groups (naive, experimental, and control) of 4-16 snails in each were performed. Each animal was used only in one series of experiments. Experimental procedures are in compliance with the Guide for the Care and Use of Laboratory Animals published by the National Institutes of Health. 

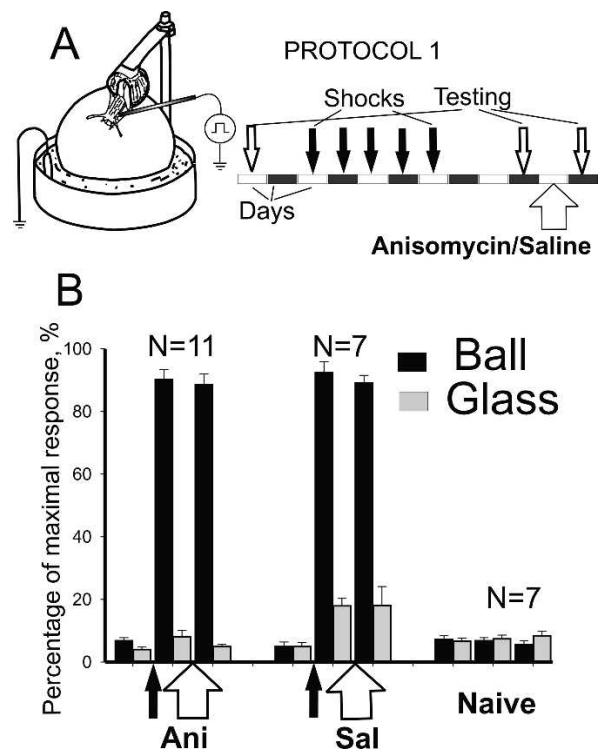

Figure 1. (A) Protocol of context conditioning experiment with anisomycin/saline injection after testing for context conditioning, no reminding. (B) Averaged amplitudes ( \pm SEM) of withdrawal responses in three groups of snails measured in two different contexts.

\section{Training and apparatus}

In the experimental set-up, the snail was tethered by its shell in a manner allowing it to crawl on a ball that rotated freely in a water containing $0.01 \% \mathrm{NaCl}$ (Fig. 1A, illustration). The ball was laced with bare stainless steel wire to complete an electrical circuit between the animal's foot and a carbon electrode placed in the water.

Electric shock was delivered by using a 1- to 4-mA, 1-sec current through a macroelectrode applied manually to the dorsal surface of the snail's foot, and the second electrode (a carbon one) was placed in the water in which the ball floated (Fig. 1A, illustration).

Punctate mechanical stimuli were applied with calibrated von Frey hairs, permitting delivery of pressures ranging from 6 (estimated as weak)-68 (noxious) $\mathrm{g} / \mathrm{mm}^{2}$. After several pilot series, the behavioral response, the intensity $\left(25 \mathrm{gm} / \mathrm{mm}^{2}\right)$, and the location of tactile stimulation were chosen. Ommathophore (posterior tentacles) withdrawal to the tactile stimulation of the rostral part of the foot skin $4-5 \mathrm{~mm}$ behind the posterior tentacles appeared to be at the level of $4 \%-20 \%$ of maximal in normal animals. In pilot experiments, it was shown that responses to such test stimulation were sensitized after noxious stimuli, and this part of the foot skin was chosen as the standard for tactile stimulation.

An investigator, blind to the experimental histories of the animals, applied the tactile stimuli to the skin of the snail and visually estimated the tentacle withdrawal amplitude in percentages, with the maximal withdrawal taken as $100 \%$. Estimation of withdrawal amplitude for each snail was "quanted": A response was qualified as $10 \%$ if only a minor withdrawal was noted and as $25 \%, 50 \%, 75 \%, 90 \%$, and $100 \%$ with increasing amplitude of withdrawal. Four to five tests per day (with 5- to 10-min intervals) were scored for each animal, and median response amplitude was calculated. In the pilot experiment, we compared this method that we used for a long time (see Balaban and Bravarenko 1993) with data obtained by using video imaging. The snail's behavior was recorded with two video cameras placed at the angle of $90^{\circ}$ to one another and connected to the computer video capture boards (Miro Video DRX). We digitized video at the reso- lution $384 \times 288$ (frame rate, 8.3 per sec), and analyzed video by using software PhysVis 1.4 (Kenyon College), which is available on the Internet. By using data from two simultaneous recordings, we calculated the three-dimensional coordinates of the tip and base of the tentacle. We analyzed the distance between tip and base of the tentacle, the tentacle tip shift per time bin $(0.12 \mathrm{sec})$, and the coordinates of the unit length direction vector. It appeared that this objective method gives uneven results due to constant changes in maximal tentacle protraction level (taken as $100 \%)$ and the head location in the space. The difference between the means of visual estimation of tentacle withdrawal amplitude by the investigator and offline quantification of responses from recordings was $\sim 5 \%$, with SEM ranging from $1 \%-$ $16 \%$ (pilot experiments in five animals, 10 measurements in each), which is less than the difference between the responses of the same snails at different trials. The results reported here were obtained by using visual estimation of tentacle withdrawal in double-blind conditions, which allowed us to increase significantly the number of animals in each series.

Before training, for $2 \mathrm{~d}$ each snail was exposed for $30 \mathrm{~min}$ daily to the experimental set-up. Then the first test session was performed for all yoked groups (first day on each protocol) (Figs. 1-4). After obtaining the pre-training scores, snails of experimental and control groups received five electrical shocks per day with 20-min intervals for $5 \mathrm{~d}$ (training). A yoked group of naive animals was necessary to estimate the possible effects of season. Current was individually chosen for each snail so that a complete withdrawal of the anterior part of the body was observed in response to a shock. No testing with tactile stimulation was performed during training days. Two days after completion of the sensitizing treatment (animals were fed during the rest period), the responsiveness to the same test tactile stimuli was compared in three yoked groups of snails (for each series of experiments) that later were labeled as naive, control, and experimental snails, in accordance with the procedure. Double-blind testing was performed at two contexts, the experimental set-up (plastic ball floating in water) and the non-reinforced context: on the flat glass lid of the terrarium in which the animals were kept between sessions. To reduce possible effects of recent handling, the test was administered no sooner than $5 \mathrm{~min}$ after the subjects had been placed in the environment. Only actively moving animals were tested. No shocks were delivered during the test sessions.

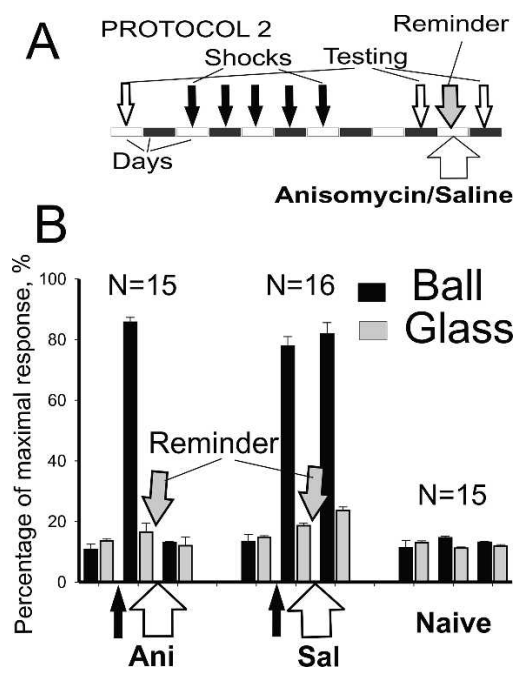

Figure 2. (A) Protocol of a context conditioning experiment with anisomycin/saline injection immediately after reminding. (B) Averaged amplitudes $( \pm$ SEM) of withdrawal responses in three groups of snails measured in two different contexts. 
A PROTOCOL 3
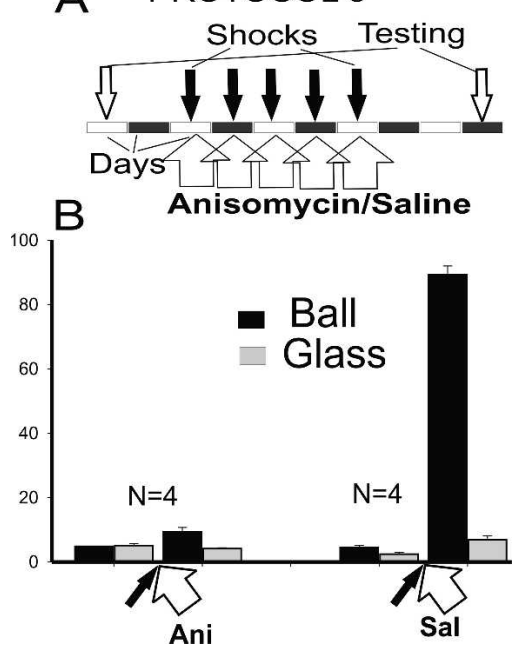

Figure 3. (A) Protocol of a context conditioning experiment with anisomycin injection immediately after each electric shock. (B) Averaged amplitudes $( \pm$ SEM) of withdrawal responses in experimental (anisomycin injection) and control (saline injection) groups of snails measured in two different contexts.

\section{Reminder}

Next day after testing, the experimental and control groups of snails were reminded of training by placing them for $20 \mathrm{~min}$ in the same context where they were shocked (ball). Immediately after the reminder (protocols 2, 4) (Figs. 2, 4), the snails were injected with either AN $(0.4 \mathrm{mg}$ in $0.2 \mathrm{~mL}$ of saline per snail weighing $30-35 \mathrm{~g}$ ) or vehicle ( $0.2 \mathrm{~mL}$ saline). As an additional control, we injected AN or vehicle to groups of snails without the reminder (protocol 1) (Fig. 1) and to a group of snails during the sensitization training (protocol 3) (Fig. 3). A yoked group of naive animals was tested on same days as were the experimental and controls for each series of experiments to control for unexpected (seasonal) changes in responsiveness.

Double-blind testing was performed on the next day after injection. Comparison was made only for yoked groups of animals. We used ANOVA for comparison of many groups, and nonparametric Mann-Whitney rank sum test was used post hoc to compare the responses of the same group of animals in two conditions.

\section{Drugs and injections}

AN (Sigma) was dissolved in sterile saline, adding an equimolar amount of $3 \mathrm{~N} \mathrm{HCl}$ and adjusting the $\mathrm{pH}$ of the resulting solution to 7.2 with $3 \mathrm{~N} \mathrm{NaOH}$. Control animals received an injection of the same volume of vehicle. Intracoelomic injection $(0.2 \mathrm{~mL})$ was performed with a fine needle via a nonsensitive part of the foot skin normally hidden inside the shell. During injection the snail stopped locomotion, mostly because the shell was fixed by the investigators, but it showed no generalized withdrawal or changes in responses to tactile stimulation.

\section{Results}

\section{Context memory in snail}

No significant difference in amplitudes of tentacle withdrawals to the test tactile stimuli was observed between all groups of snails either on the plastic ball or on a flat glass surface during pre-training testing (ANOVA was used) (see first two columns for each group on Figs. 1-4).

After testing, the experimental and control groups were sub- jected to electric shock for $5 \mathrm{~d}$ (see protocols on each figure), and after $2 \mathrm{~d}$ of rest, the animals were tested in both environments. Averaged results indicate that in all cases the responses in the context in which the snails were shocked were significantly bigger than those in the neutral context $(P<0.001$, Mann-Whitney) ( $n$ for each group is shown on Figs. 1,2 ). The difference in responses of a yoked naive group of snails in all contexts was not significant (Figs. 1, 2, 4). It means that the snails displayed an increase in amplitude defensive reaction only in the environment that had a history of shocks' delivery. This outcome is consistent with the assumption that the snails can differentiate the environment in which shocks were given. The context specificity of that enhancement is extremely important because it allows us to rule out a sensitizing effect of the shock as the sole result of sensitization training. These data suggest the existence of long-term changes in behavior (appearance of a contextdependent memory) after noncontingent noxious stimulation that can be observed only in the context in which the stimulation was applied.

\section{Effects of AN injection on context memory}

To check whether the injections of AN itself may influence a context memory, we injected trained experimental snails with AN the next day after the memory testing, as well as a control group with saline without reminding of the context in which the sensitization training was performed. No significant differences in responses were observed in snails injected with AN or vehicle (saline) in these experiments (Fig. 1). A significant difference in responses $(P<0.001$, Mann-Whitney) ( $n$ is shown on Fig. 1$)$ was found under both conditions in behavioral responses to test stimuli in the context in which the snails were shocked and in the neutral context. The results suggest that the AN injection by itself does not impair the context memory.

In the next series of experiments, we tested whether reminding of the context can influence formed memory, and it will become sensitive to protein synthesis blocker. It was observed that the responses of the snails AN injected after the reminder (placing them on the ball where sensitization training was performed) and tested next day were not different in both contexts,

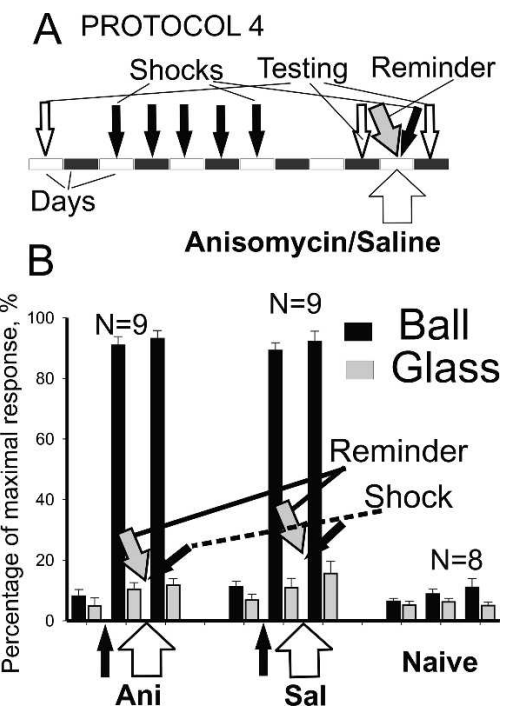

Figure 4. (A) Protocol of a context conditioning experiment with the anisomycin injection immediately after the combined reminding and sensitization stimuli. $(B)$ Averaged amplitudes ( \pm SEM) of withdrawal responses in three groups of snails measured in two different contexts (ANOVA, $P=0.45$ ).

\section{Learning \& Memory




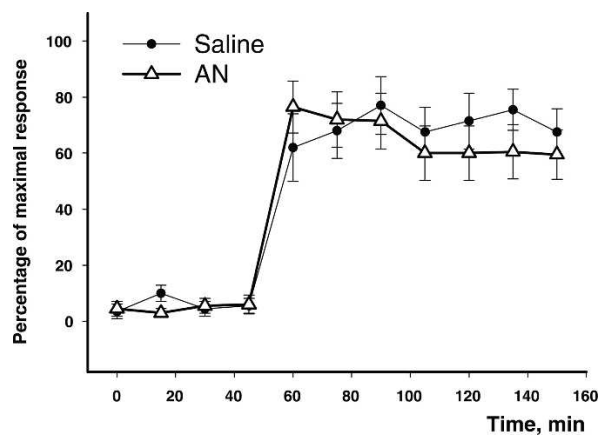

Figure 5. Averaged changes in amplitude of behavioral responses in two groups of snails ( $n=6$ in each group) injected $10 \mathrm{~min}$ prior to beginning of testing with AN or saline. After four test stimuli, one electric shock (timing is marked by an arrow) was delivered to the skin and testing continued. No significant difference in responses of snails from two groups was observed (ANOVA, $P=0.4$ ), which suggests that AN does not influence short-term (tens of minutes) changes in excitability.

while responses of snails from a yoked group injected similarly by vehicle (saline) differed significantly in different contexts $(P<0.001$, Mann-Whitney) ( $n$ is shown on Fig. 2$)$. These results suggest that the AN injection combined with reminding impaired the formed context memory or its retrieval. Certain differences in initial levels of responses in the series of experiments shown in Figures 1 and 2 may be due to season or a new stock of animals brought from nature.

As it was shown previously, just application of AN was not able to block the consolidated context memory (Fig. 1). It was of interest for us to find out whether $\mathrm{AN}$ can influence context memory at the stage of its consolidation in snails, and in the next series of experiments, we injected AN 3-5 min after the first sensitization stimulus (the effect of AN lasted during the whole training session, 1-2 h). The procedure was repeated for $5 \mathrm{~d}$ (see protocol on Fig. 3). No differences in responses to test stimuli in both contexts were found in the AN-injected snails, while in the yoked control group, the difference was highly significant (Fig. 3 ). Results of these experiments (Fig. 3) indicate that AN applied at a stage of context memory consolidation prevents formation of long-term associative changes in behavior.

\section{Interference of reinforcing stimuli}

It is well known that a strong reinforcing stimuli can conceal effects of other stimuli and interfere with ongoing processes (Pavlov 1927). In the next series of experiments, we tested whether the reinforcement during the reminding session would change the effects of reminding. On the next day after the test session that followed the training session, the snails were reminded of context in which they were shocked and received two additional reinforcing stimuli, after which the snails were injected with AN (see protocol on Fig. 4A). Testing of effects was performed on the following day. Averaged results indicate that vehicle-injected snails behave similarly to AN-injected animals (Fig. 4B). Thus, application of the reinforcing stimuli interferes with the effects of reminding.

Specificity of the AN effects for the long-term memory To check whether the AN injections influence short-term nonassociative plasticity, we performed a series of experiments in which the responses to test tactile stimuli were delivered at 15min intervals, and one ( $2 \mathrm{sec}, 2-4 \mathrm{~mA}$ ) electric shock was given. Normally after such a shock, the amplitude of behavioral responses increased for 1-2 h. Averaged results showed (Fig. 5) that behavioral responses in the snail groups that were injected with
AN or saline prior to the beginning of testing were not different (ANOVA, $P=0.4$ ). These results suggest that the AN injection by itself does not influence the short-term changes in excitability elicited by the facilitating stimuli.

To demonstrate a specific effect of AN on the consolidation of long-term memory, it is critical to demonstrate intact shortterm memory. We performed an independent series of experiments in which the existing stable context memory was tested 4 $\mathrm{h}$ and $24 \mathrm{~h}$ after the reminder $+\mathrm{AN} /$ saline injection (Fig. 6). It was observed that the responses of the snails in all four groups tested $4 \mathrm{~h}$ after the reminder/no-reminder $+\mathrm{AN} /$ saline injection were not different to the responses in the same context $2 \mathrm{~d}$ earlier (ANOVA, $P=0.21$ ), while the difference in responses in two contexts was still significant (responses in two contexts were compared, Mann-Whitney, $P<0.001$ ) ( $n$ is shown on Fig. 6). Testing $24 \mathrm{~h}$ after the treatment + injection showed that a significant loss of memory (ANOVA with post hoc analysis, $P<0.01$ ) was observed only after the reminder + AN injection. These results suggest that the AN injection combined with the reminding specifically impaired long-term context memory or its retrieval.

\section{Discussion}

Memory reactivation is dependent on protein synthesis Described results confirm the existence of associative context memory in terrestrial snails (as was shown earlier in Balaban and Bravarenko 1993) and show that reactivation of memory (reminding) $2 \mathrm{~d}$ after development of context memory elicits protein synthesis-dependent processes, involved either in memory storage or retrieval.

In addition to our early article, only two accounts on context memory in snails were published: a study of context conditioning in Aplysia (Colwill et al. 1988) and a recent study of context learning in Lymnaea (Haney and Lukowiak 2001). The phenomenon of reconsolidation of aversive conditioning in slugs was shown previously (Sekiguchi et al. 1997). In an article by Pedreira et al. (2002), reactivation and reconsolidation of con-

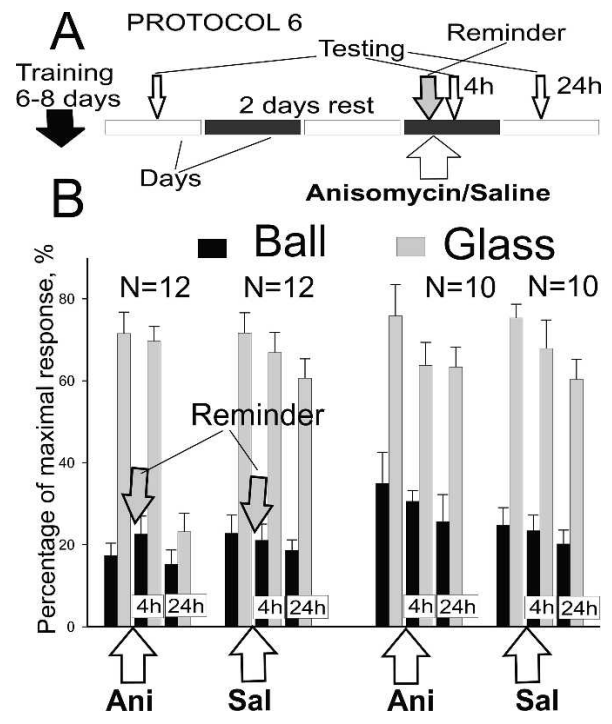

Figure 6. Specificity of the anisomycin effects for the long-term memory. Context conditioning experiment with testing of memory 4 and $24 \mathrm{~h}$ after the reminder + anisomycin/saline injection. (A) Protocol of the experiment. (B) Averaged amplitudes ( \pm SEM) of withdrawal responses in four groups of snails measured in two different contexts. Significant loss of memory (ANOVA with post hoc analysis, $P<0.01$ ) was observed only $24 \mathrm{~h}$ after the reminder + anisomycin injection. 
text memory in the crab Chasmagnathus was described. Basically, the results obtained in crabs are similar to those reported here, but there is a difference in analyzed memory that may be of importance. It was shown that snails need at least 5-6 d to learn the difference in the two contexts, and this memory lasts at least 2 wk (Balaban and Bravarenko 1993). After the one-session learning, same as used in crabs by Pedreira et al. (2002), in terrestrial snails only an analog of the long-term sensitization nonspecific to used stimuli was observed. Similar long-term sensitization was thoroughly investigated in Aplysia (Cleary et al. 1998). Terrestrial snails after the one-session aversive training reacted with increased withdrawal responses to any noxious stimuli or potentially dangerous contexts for 1-2 d only. In Pavlovian terminology it is called a "nonspecific generalized reflex," which accompanies any conditioning at the early stage (Pavlov 1927). Only after 5-6 d of associating the context and reinforcement, and a period of rest (1-2 d) do the snails (and dogs in Pavlov experiments) display in full a context conditioning, indicating that a specific context memory is formed. It was the reason why we used such a protocol in our experiments, and it is our opinion that the multisession memory may be quite different from the one-session learning. We do not argue that mammals can achieve some memory in $1 \mathrm{~d}$, but snails and Pavlov dogs have to experience the stage of "generalized conditioning" and only after at least several days of learning do they form a "specialized conditioned response," i.e., a stable long-term associative memory. Present experiments showed that administration of AN during the learning session blocked formation of the context memory completely (Fig. 3), while similar administration of AN in an animal with fully formed context memory (specialized conditioned reflex) after additional reinforcing stimulus in the same context had no effect on memory (Fig. 4). It suggests that the protein synthesis is necessary for new memory but does not influence significantly the memory that was formed previously. Similar findings were shown in a study by Eisenberg and Dudai (2004) on fish. They showed that "old fears," i.e., memory already lasting days, in fish become resistant over time to postreactivation interference.

With this background, the experiments with reminding and clear effects of AN specific for long-term memory suggest that, in this case, protein synthesis interferes not with the long-term memory (lasting 6-8 d) but rather with a renewal of retrieval. In snails with formed long-term contextual memory, it was impaired by injection of AN specifically after the reminding (cf. Figs. 1 and 2), which suggests either deficit of retrieval of memory or impairment of consolidated memory due to reconsolidation elicited by reminding. Additional experiments should be made to distinguish between these two possibilities, but our present data suggest that reconsolidation elicited by reminding affects ways of memory retrieval, not memory. Recent experiments by Duvarci and Nader (2004) suggest that reconsolidation, but not retrieval, is affected by AN. Unfortunately, one-trial learning in these experiments was used, which we consider significantly different from classical Pavlovian learning, which requires "repetition" of the learning situation for at least several days (Pavlov 1927).

Recently, it was shown (Lattal and Abel 2004) that the impairments in behavior caused by the protein synthesis inhibitor AN given after retrieval are temporary and are thus not likely to reflect disruptions in a protein synthesis-dependent memory reconsolidation process. When the AN injections in those experiments followed acquisition, freezing was impaired during memory tests the next day and $21 \mathrm{~d}$ later. When the AN injections followed normal retrieval of contextual fear conditioning, freezing was impaired the next day but recovered to the levels of control mice when testing occurred $21 \mathrm{~d}$ later. The recovery effect was specific to the conditioning context. These results suggest that the AN injections after retrieval do not retroactively affect the memory from conditioning. More and more investigators agree that impairment of memory after retrieval can be different from impairment of memory after acquisition (Taubenfeld et al. 2001; Anokhin et al. 2002; Eisenberg and Dudai 2004). Absence of the AN-induced effects when applied after reminding combined with reinforcement (Fig. 4) conforms to the latter suggestion. Similar results were obtained in fish (Eisenberg and Dudai 2004).

\section{Procedural and contextual memory}

There is no clear distinction in the literature of different neural mechanisms underlying procedural and context memory, although there is an agreement that contextual memories may be stored in a separate structure (Thomas et al. 2002). In our previous experiments in snail Helix with selective ablation of serotonergic neurons with a neurotoxin 5,7-DiHT before and after development of aversive conditioning, it was shown that serotonergic neurons are necessary for formation of procedural memory only during the consolidation phase, but they are not necessary for recall. Quite different results, demonstrating that serotonergic neurons are necessary for formation, recall, and/or retention, were obtained in experiments concerning environmental (context) conditioning (Balaban 2002a,b). Fully formed context memory was impaired by suppression of the serotonergic system in learned animals, thus suggesting that the serotonincontaining cells are involved in retrieval and maintenance of this acquired behavior. Independence of the cellular mechanisms involved suggests that during learning the animal acquires information about the context in which it receives the reinforcement and independently stores information about certain context stimuli, which are contingent on reinforcement. Thus, the animals can perceive the same conditioned stimulus in another context as a novel one, and are prepared in the known context to respond to noxious stimuli. The experiments reported here with AN blockade of context memory may be interpreted at the cellular level as interference in the long-term plasticity of synaptic inputs of serotonergic neurons, and we plan to explore neural correlates of those changes.

\section{Acknowledgments}

The work was supported in part by grants from the Russian Foundation for Basic Research (04-04-48817, 05-04-48724) and INTAS grant 01-2117.

\section{References}

Anokhin, K.V., Tiunova, A.A., and Rose, S.P. 2002. Reminder effects: Reconsolidation or retrieval deficit? Pharmacological dissection with protein synthesis inhibitors following reminder for a passive-avoidance task in young chicks. Eur. J. Neurosci. 15: $1759-1765$.

Antonov, I., Antonova, I., Kandel, E.R., and Hawkins, R.D. 2003. Activity-dependent presynaptic facilitation and hebbian LTP are both required and interact during classical conditioning in Aplysia. Neuron 37: 135-147.

Balaban, P.M. 1993. Behavioral neurobiology of learning in terrestrial snails. Prog. Neurobiol. 41: 1-19.

. 2002a. Declarative and procedural memory in animals with simple nervous systems. In Psychology at the turn of the millennium (ed. C. Hofsten), pp. 1-28. Academic Press, Stockholm.

. 2002b. Cellular mechanisms of behavioral plasticity in terrestrial snail. Neurosci. Biobehav. Rev. 26: 597-630.

Balaban, P.M. and Bravarenko, N.I. 1993. Long-term sensitization and environmental conditioning in terrestrial cells. Exp. Brain Res. 96: $487-493$.

Balaban, P.M., Korshunova, T.A., and Bravarenko, N.I. 2004. Postsynaptic calcium contributes to reinforcement in a three-neuron network exhibiting associative plasticity. Eur. J. Neurosci. 19: $227-233$.

\section{Learning \& Memory}


Cleary, L.J., Lee, W.L., and Byrne, J.H. 1998. Cellular correlates of long-term sensitization in Aplysia. J. Neurosci. 18: 5988-5998.

Colwill, R.M., Absher, R.A., and Roberts, M.L. 1988. Context-US learning in Aplysia californica. J. Neurosci. 8: 4434-4439.

Debiec, J. and LeDoux, J.E. 2002. Cellular and systems reconsolidation in the hippocampus. Neuron 36: 527-538.

Duvarci, S. and Nader, K. 2004. Characterization of fear memory reconsolidation. J. Neurosci. 24: 9269-9275.

Eisenberg, M. and Dudai, Y. 2004. Reconsolidation of fresh, remote, and extinguished fear memory in medaka: Old fears don't die. Eur. J. Neurosci. 20: 3397-3403.

Ghirardi, M., Benfenati, F., Giovedi, S., Fiumara, F., Milanese, C., and Montarolo, P.G. 2004. Inhibition of neurotransmitter release by a nonphysiological target requires protein synthesis and involves cAMP-dependent and mitogen-activated protein kinases. J. Neurosci. 24: $5054-5062$.

Haney, J. and Lukowiak, K. 2001. Context learning and the effect of context on memory retrieval in Lymnaea. Learn. Mem. 8: 35-43.

Lattal, K.M. and Abel, T. 2004. Behavioral impairments caused by injections of the protein synthesis inhibitor anisomycin after contextual retrieval reverse with time. Proc. Natl. Acad. Sci. 101: $4667-4672$.

Misanin, J.R., Miller, R.R., and Lewis, D.J. 1968. Retrograde amnesia produced by electroconvulsive shock after reactivation of a consolidated memory trace. Science 160: 554-555.

Nader, K., Schafe, G.E., and Le Doux, J.E. 2000. Fear memories require protein synthesis in the amygdala for reconsolidation after retrieval. Nature 406: 722-726.

Pavlov, I.P. 1927. Conditioned reflexes, and investigation of the physiological activity of the cerebral cortex. Oxford Press, Oxford, UK.

Pedreira, M.E., Perez-Cuesta, L.M., and Maldonado, H. 2002. Reactivation and reconsolidation of long-term memory in the crab Chasmagnathus: Protein synthesis requirement and mediation by NMDA-type glutamatergic receptors. J. Neurosci. 22: 8305-8311.

Sangha, S., Scheibenstock, A., and Lukowiak, K. 2003. Reconsolidation of a long-term memory in Lymnaea requires new protein and RNA synthesis and the soma of right pedal dorsal 1. J. Neurosci. 23: 8034-8040.

Sara, S.J. 2000. Retrieval and reconsolidation: Toward a neurobiology of remembering. Learn. Mem. 7: 73-84.

Sekiguchi, T., Yamada, A., and Suzuki, H. 1997. Reactivation-dependent changes in memory states in the terrestrial slug Limax flavus. Learn. Mem. 4: 356-364.

Taubenfeld, S.M., Milekic, M.H., Monti, B., and Alberini, C.M. 2001. The consolidation of new but not reactivated memory requires hippocampal C/EBPß. Nat. Neurosci. 8: 813-818.

Thomas, K.L., Hall, J., and Everitt, B.J. 2002. Cellular imaging with zif268 expression in the rat nucleus accumbens and frontal cortex further dissociates the neural pathways activated following the retrieval of contextual and cued fear memory. Eur. J. Neurosci. 16: $1789-1796$.

Zakharov, I.S., Ierusalimsky, V.N., and Balaban, P.M. 1995. Pedal serotonergic neurons modulate the synaptic input of withdrawal interneurons in Helix. Invert. Neurosci. 1: 41-52.

Received June 8, 2005; accepted in revised form September 9, 2005. 


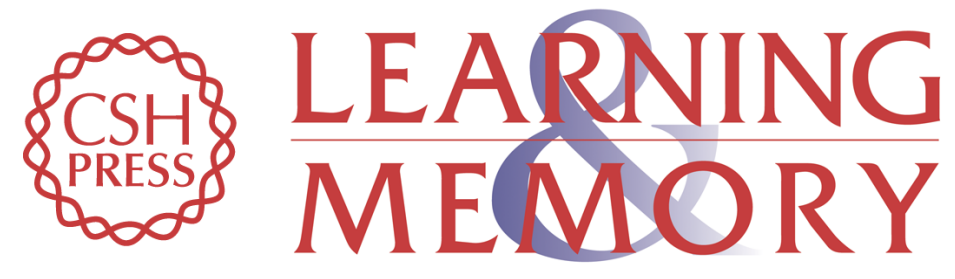

\section{Reconsolidation of a context long-term memory in the terrestrial snail requires protein synthesis}

Tatiana H. Gainutdinova, Rosa R. Tagirova, Asja I. Ismailova, et al.

Learn. Mem. 2005, 12:

Access the most recent version at doi:10.1101//m.25705

References This article cites 23 articles, 11 of which can be accessed free at:

http://learnmem.cshlp.org/content/12/6/620.full.html\#ref-list-1

License

Email Alerting Receive free email alerts when new articles cite this article - sign up in the box at the Service top right corner of the article or click here. 